\title{
Video Interviewing: An exploration of the feasibility as a mode of survey application
}

McKinlay Jeannis, ${ }^{\star}$ Tamara Terry ${ }^{\dagger}$, Richard Heman-Ackah, Michael Price

Keywords: survey, data collection, skype, rti, facebook, video interviewing

DOI: $10.29115 /$ SP-2013-0001

\section{Survey Practice}

Vol. 6, Issue 1, 2013

Video Interviewing: An exploration of the feasibility as a mode of survey application

To stay current with today's constant evolution of technology, survey researchers continue to seek and modernize their data collection methods and offer alternative opportunities for respondents to participate. More traditional survey data collection modes such as mail, field, telephone, and Web surveys are becoming more limited as a result of advances in communications technology. These traditional data collection methods continue to be valuable, although innovative technologies in communication add promising features and capabilities. Researchers should continue to investigate new methodologies to gain further insight on the benefits and challenges of these new technologies.

RTI's Division for Research Services is evaluating video interviewing as a new methodology in the data collection toolkit. Video communications which includes interviewing or conferencing is a steadily growing concept of Internet-based communication and is used in various industries for enhancing visual and audio communication experience.

Most desktops, laptops, tablets, and smartphones have built-in or supplemental Web cameras and microphones that allow video communications between two parties whether stationary or on the go. Video interviewing would also benefit from recording and analyzing both verbal and nonverbal communication between interviewer and respondent.

Video communications are offered through various social networking sites that many potential survey respondents check and update frequently. As the popularity of video communication increases, we focus our concept of video interviewing on two prominent social networking platforms, Facebook and Skype.

\footnotetext{
* Institution: RTI International

† Institution: RTI International

$\ddagger$ Institution: RTI International

** Institution: RTI International
} 
Founded in 2003, Skype is communications software that allows users to make phone calls using voiceover IP (VoIP) technology, video calls, and instant message chatting. With over 100 million users and 65 million daily users (C aukin 2011), Skype has become a viable communications platform. In July 2011, Facebook announced an agreement that integrated Skype's video calling feature into Facebook chat ( $\mathrm{Su} 2011)$.

Since 2004, Facebook has evolved and continued their efforts to become a legitimate supplemental, and in some cases, outright alternative, communication platform through several tools of communication: content sharing, status updates, commenting, liking, blogging, and chatting. In fact, former Facebook president Sean Parker acknowledged one clear objective of Facebook early on was to become a new communications platform (Kirkpatr ick 2011). With staggering growth rates that have reached a current monthly active user base of over 1 billion individuals (Facebook Inc 2013), and a United States and Canada penetration of nearly 20 percent, Facebook has solidified its position as a sustainable communication platform.

For video interviewing to successfully function as a method of data collection, both the interviewer and respondent's hardware and software equipment will need to meet standard requirements for successful video communication. Video communication can be a relatively inexpensive proposition. Web cameras typically range in price from 20 to 50 dollars; however, many devices already have this hardware built in. Internet service, an additional cost, would average 20 dollars a month. Most households already have Internet service on multiple devices. As reported by the Pew Research Center (2013), 82 percent of American adults use the internet, while the increase of wireless-only households has soared above 26 percent (Blumberg et al. 2011). This increase demonstrates the growing trend toward adopting new communication platforms and is a sign that similar shifts in data collection modes could be expected.

Video interviewing poses some unique capabilities, such as the ability to visually authenticate or confirm the sample member and the utilization of qualitative data to analyze nonverbal respondent cues to questions. Other visual considerations are the physical and professional appearance of the interviewing staff and whether being able to view the background of the data collection facility or respondent location compromises privacy and/or security. Last, providing visual aids to respondents further enhances communication on survey projects that rely on recall of past events or exposure.

The research on video interviewing is limited thus far. The next section reviews case studies that utilize a variation of visual interactions to provide further insight on potentially using video interviewing via Skype and Facebook to collect data. Some survey biases to consider are sample frame bias, social desirability bias, avidity bias, and non-response bias. A face-to-face interview is the closest comparison to a video-interviewing data collection method. During 
our research, we found various case studies that support face-to-face interviewing as a common, preferred method for data collection. The following case studies also show the potential benefits, areas of concern, and feasibility of video interviewing for data collection.

The purpose of the first case study was to identify problematic interviewee behaviors such as social desirability during visual interactions. This particular case study was a face-to-face interview; however, face-to-face interviews share the same concept as video interviewing as they both allow visual interaction between the interviewer and respondent. The study design reviewed 22 transcripts from three studies comprising 316 pages of the verbatim account of words and behavioral cues from a face-to-face interview, such as laughing, crying, sighing, or hesitating. Results from this study showed very little evidence of problematic respondent behavior (Collins, Shattell, and Thomas 2 005) during face-to-face interviews. We could expect the same results for video interviewing because the visual aspect from the interviewer and respondent could arguably be compared in both types of interviews. This case study supports using video interviewing as a new technology for data collection.

The second case study examined the gender-of-interviewer effects in a pre-recorded video-enhanced Web survey that asked sensitive questions about relationships and sexual experiences. This case study also utilizes the concept of visual interaction, which can be compared to video interviewing. Subjects in the study were assigned one of two conditions: a traditional text-based Web survey or an interactive Web questionnaire with embedded flash clips. Results from this study showed evidence of gender-of-interviewer effects with pre-recorded video clips of interviewers reading questions and evidence of social desirability bias in an opposite gender interview (Fuchs 2009). Similar to field studies and its direct visual interaction with respondents, video interviewing could be susceptible to social desirability bias.

The purpose of our third case study was to examine the use of Skype as a tool to assess functionality in orthopedic research and determine an easier method of patient follow-up. The study design included patients treated surgically for a shoulder injury from July 2005 to February 2010. Patients were assessed twice: once during their normal outpatient department and once via Skype within 2 weeks of the outpatient review. Results from this study showed that 92 percent of the patients had a computer and Internet access, 66 percent were existing Skype users, and 83 percent were happy to take part in a Skype assessment. Of those who were happy to take the Skype assessment, 93 percent preferred the use of Skype for follow-up due to convenience and cost. This study also confirmed that Skype can be used as a patient follow-up method (Good et al. 2 012). Overall, this case study supported that video communication technology via Skype is becoming more popular in research and that people from all demographics are using Skype as a means to communicate. This could also be a foundation for video interviewing as a viable option for survey research as more 
people are receptive to using new communications technology.

Our last case study used Facebook as a locating tool in a longitudinal study with a goal of increasing non-response in a traditional telephone study. The study design included searching for 919 non-respondents through two modes: field locating and Facebook locating. Results from this study showed that 32 percent of the non-respondents were found on Facebook and were sent messages. Of that group, 31 percent received a Facebook message and then completed the interview, eliminating the need to follow-up with a portion of non-respondents. Ultimately, 73 percent of the original non-respondents found on Facebook completed an interview for the study, which represented a 4 percent increase in total completed interviews. The additional completed cases by non-respondents were accounted to sample members seeing the Facebook message (Rhodes and Marks 2011). As mentioned earlier, one of the video-interviewing platforms we focus our research on is Facebook, which would provide an added value in not only video interviewing, but also locating sample members. This case study encourages the use of Facebook for video interviewing, finding sample members, decreasing non-response bias, and increasing response rates.

The goal of our presentation was to assess the feasibility of video interviewing in survey research, but we recognize many steps are needed to make this concept a reality. The use of video interviewing could present some advantages for researchers such as an additional medium of contact, visual authentication of sample members, potential increased response rates, and decreased non-response bias. With the increase of cell phone only households, offering video interviewing as another medium of communication could add value to data analysis and sample member participation. Our next steps are (1) identify and conduct a pilot test (2) design and create methodological protocols, (3) obtain RTI's Institutional Review Board approval, and (4) purchase necessary equipment. 


\section{REFERENCES}

Blumberg, S.J., J.V. Luke, N. Ganesh, M.E. Davern, M.H. Boudreaux, and K. Soderberg. 2011. "Wireless Substitution: State-Level Estimates." National Health Statistics Reports 39: 1-28. http://www.cdc.gov/nchs/data/nhsr/nhsr039.pdf.

Caukin, J. 2011. “A Day in the Life of Skype \#Infographic.” Big Blog Skype (blog). September 6, 2011. http://blogs.skype.com/2011/09/06/a-day-in-the-life-of-skype-inf/\#fbid=FBV3oJZ-pQ.

Collins, M., M. Shattell, and S.P. Thomas. 2005. "Problematic Interviewee Behaviours." Western Journal of Nursing Research 27 (2): 188-99. http://wjn.sagepub.com/content/27/2/188.

Facebook Inc. 2013. "User Metrics 1.” U.S. Securities and Exchange Commission. http://www.sec.gov/Archives/edgar/data/1326801/000132680113000003/usermetrics1.jpg.

Fuchs, M. 2009. "Gender-of-Interviewer Effects in a Video-Enhanced Web Survey: Results from a Randomized Field Experiment.” Social Psychology 40: 37-42.

Good, D.W., D.F. Lui, M. Leonard, S. Morris, and J.P. McElwain. 2012. "Skype: A Tool for Functional Assessment in Orthopaedic Research." Journal of Telemedicine and Telecare Online First, 1-5.

Kirkpatrick, D. 2011. The Facebook Effect: The inside Story of the Company That Is Connecting the World. New York: Virgin Publishing.

Pew Research Center. 2013. "Internet Use and Home Broadband Connections: Demographics.” Pew Internet. http://www.pewinternet.org/Infographics/2012/Internet-Use-and-HomeBroadband-Connections.aspx.

Rhodes, B.B., and E.L. Marks. 2011. “Using Facebook to Locate Sample Members.” Survey Practice. http://surveypractice.wordpress.com/2011/10/24/using-facebook-to-locate-samplemembers.

Su, P. 2011. "Call Your Friends Right from Facebook.” The Facebook Blog (blog). 2011. http://blog.facebook.com/blog.php?post=10150223135777131. 